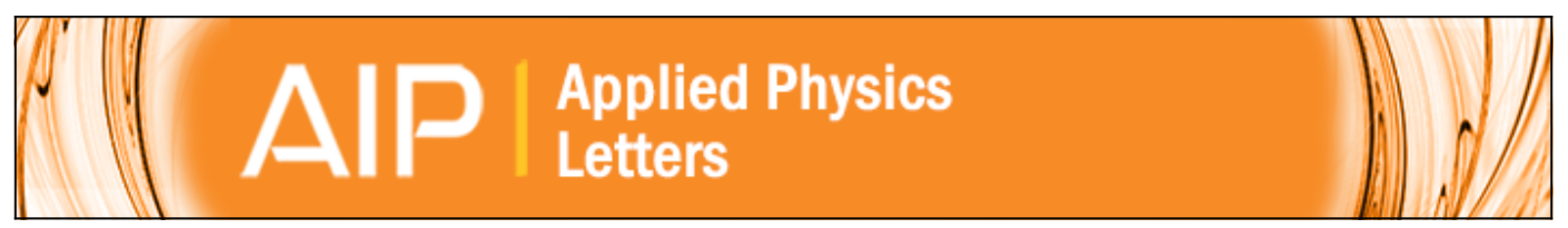

\title{
Photocurrent extraction efficiency in colloidal quantum dot photovoltaics
}

K. W. Kemp, C. T. O. Wong, S. H. Hoogland, and E. H. Sargent

Citation: Applied Physics Letters 103, 211101 (2013); doi: 10.1063/1.4831982

View online: http://dx.doi.org/10.1063/1.4831982

View Table of Contents: http://scitation.aip.org/content/aip/journal/apl/103/21?ver=pdfcov

Published by the AIP Publishing

\section{Articles you may be interested in}

Charge transport and recombination in $\mathrm{P} 3 \mathrm{HT}: \mathrm{PbS}$ solar cells

J. Appl. Phys. 117, 095503 (2015); 10.1063/1.4913952

Systematic optimization of quantum junction colloidal quantum dot solar cells

Appl. Phys. Lett. 101, 151112 (2012); 10.1063/1.4757866

Organic/inorganic hybrid pn-junction between copper phthalocyanine and CdSe quantum dot layers as solar cells J. Appl. Phys. 112, 044507 (2012); 10.1063/1.4747835

Ultraviolet electroluminescence from colloidal $\mathrm{ZnO}$ quantum dots in an all-inorganic multilayer light-emitting device

Appl. Phys. Lett. 100, 061104 (2012); 10.1063/1.3682307

Nanowire-quantum-dot solar cells and the influence of nanowire length on the charge collection efficiency

Appl. Phys. Lett. 95, 193103 (2009); 10.1063/1.3258490

\section{AIP $\mid$ APL Photonics}

APL Photonics is pleased to announce Benjamin Eggleton as its Editor-in-Chief

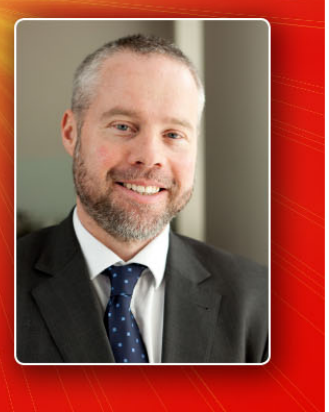




\title{
Photocurrent extraction efficiency in colloidal quantum dot photovoltaics
}

\author{
K. W. Kemp, C. T. O. Wong, S. H. Hoogland, and E. H. Sargent \\ Department of Electrical and Computer Engineering, University of Toronto, 10 King's College Road, \\ Toronto, Ontario M5S 3G4, Canada
}

(Received 9 August 2013; accepted 1 November 2013; published online 18 November 2013)

\begin{abstract}
The efficiency of photocurrent extraction was studied directly inside operating Colloidal Quantum Dot (CQD) photovoltaic devices. A model was derived from first principles for a thin film p-n junction with a linearly spatially dependent electric field. Using this model, we were able to clarify the origins of recent improvement in CQD solar cell performance. From current-voltage diode characteristics under 1 sun conditions, we extracted transport lengths ranging from $39 \mathrm{~nm}$ to $86 \mathrm{~nm}$ for these materials. Characterization of the intensity dependence of photocurrent extraction revealed that the dominant loss mechanism limiting the transport length is trap-mediated recombination. (C) 2013 AIP Publishing LLC. [http://dx.doi.org/10.1063/1.4831982]
\end{abstract}

Solution-processed colloidal quantum dot (CQD) photovoltaics have progressed significantly in their performance in recent years, with certified results now exceeding $7 \% .{ }^{1}$ As a result of these advances in performance, there is a growing need to understand the basic electronic material properties that govern recombination and transport.

It is well established that in CQD photovoltaics today, there exists an absorption-extraction compromise ${ }^{2,3}$ wherein device thickness, and therefore absorption, cannot be increased to the point of full solar spectrum optical absorption without compromising the ability to extract photocharges efficiently. This compromise limits further progress in device performance, not only as it pertains to short-circuit current Jsc but also in light of the voltage-dependent extraction of drift-transported photocharges, a reliance that degrades fill factor. To study the origins of this behaviour, we measured photocarrier collection efficiency in recently developed CQD materials. ${ }^{1}$

We examined the organically cross-linked and hybridpassivated CQD solar cell each reported previously. ${ }^{1}$ It was found that additional $\mathrm{CdCl}_{2}$ treatment of the $\mathrm{PbS}$ nanocrystals led to substantially improved photovoltaic performance from $5.6 \%$ to $7.4 \%$ efficiencies for the organically cross-linked and hybrid-passivated CQD materials, respectively. Much of this enhancement can be attributed to the improvement in $\mathrm{J}_{\mathrm{SC}}$ from 17 to $22.8 \mathrm{~mA} \mathrm{~cm}^{-2}$.

The experimental collection efficiencies (Figures 1(b) and 1(c)) for the organically cross-linked and hybridpassivated CQD devices, respectively, were obtained by measuring the intensity-dependent current-voltage relationship. Devices were fabricated using previously established methods. ${ }^{1}$ An $830 \mathrm{~nm}$ fiber-coupled laser diode was used as an excitation source and a neutral density filter wheel was used to control the light intensity reaching the device. By measuring $\mathrm{J}-\mathrm{V}$ data at two different intensities, we calculate the experimental collection efficiency ${ }^{4}$ from

$$
\eta(V)=\operatorname{IQE}(\lambda) \frac{J_{1}(V)-J_{2}(V)}{J_{S C_{1}}-J_{S C_{2}}},
$$

where IQE is the internal quantum efficiency at the excitation wavelength and $\mathrm{J}_{1,2}(\mathrm{~V})$ and $\mathrm{J}_{\mathrm{SC} 1,2}$ are the photocurrent density and short circuit current density at a given excitation intensity.

Extracting useful insights from the experimental data requires the development of an appropriate model which takes into account the physical and electronic properties of CQD solar cells. The work builds on previously reported models of voltage-dependent charge extraction in solar cells. ${ }^{5-7}$

One of the simplest models (Figure 2(a)) used to explain collection efficiency in solar cells is the Gärtner expression, ${ }^{8}$ which assumes that carriers are extracted within one diffusion length, $\mathrm{L}_{\mathrm{D}}$ of the depletion region thickness, $\mathrm{W}(\mathrm{V})$ as

$$
\eta(V)=1-\frac{\exp (-\alpha W(V))}{1+\alpha L_{D}},
$$

where $\alpha$ is the absorption length in the material and $\mathrm{V}$ is the applied bias.

This model assumes perfect extraction of carriers generated within the depletion region. In reality, losses may exist even in the presence of a strong electric field in the scenario in which charge carriers cannot be swept out prior to recombination. Prior reports confirm that this situation exists in certain CQD solar cells, since there exist previously reported devices that, though fully depleted, exhibited IQE well below $80 \%$. $^{2,9,10}$

Extraction of charge carriers undergoing drift transport in an electric field has been previously modeled (Figure 2(b)) using the Hecht equation ${ }^{6}$

$$
\eta(V)=\frac{\mu \tau_{r e c}\left(V_{B}-V\right)}{d^{2}}\left[1-\exp \left(\frac{-d^{2}}{\mu \tau_{r e c}\left(V_{B}-V\right)}\right)\right],
$$

where $V_{B}$ is the built in voltage, $d$ is the device thickness, $V$ is the forward bias, and $\mu \tau_{\text {rec }}$ is the mobility-lifetime product. The Hecht equation depends on the ratio of the transit time, $\tau_{\mathrm{t}}$, and recombination lifetime, $\tau_{\text {rec }}$, indicated in Figure 2(b) where $\tau_{\mathrm{t}}$ is given by

$$
\tau_{t}=\frac{d^{2}}{\mu\left(V_{B}-V\right)} .
$$


(a)

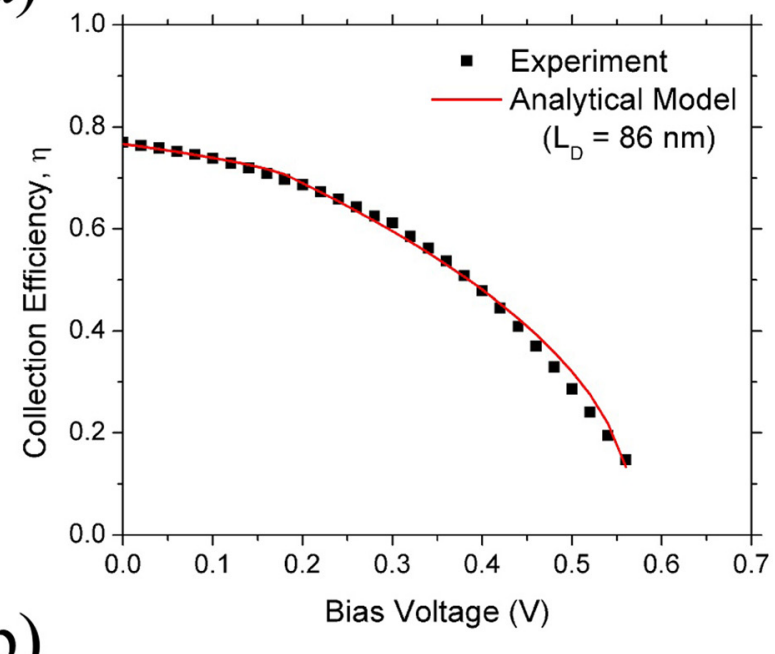

(b)

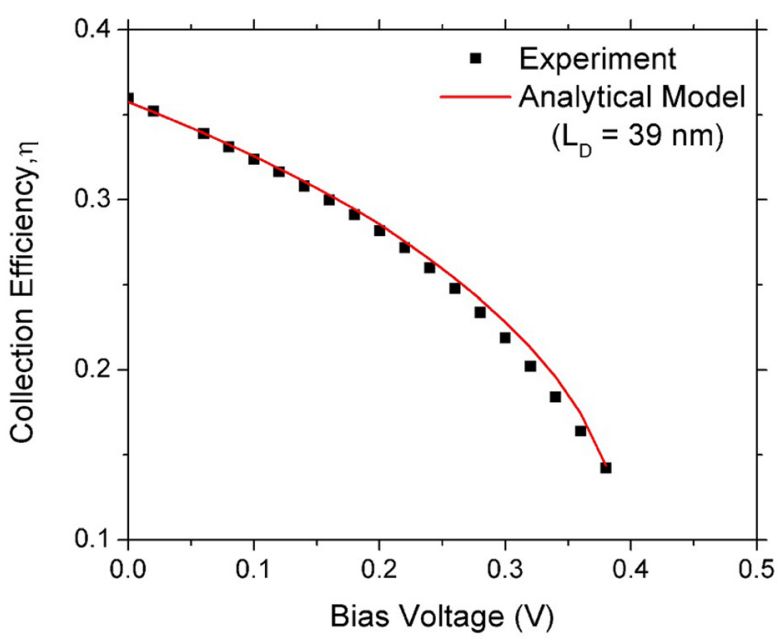

FIG. 1. Collection efficiency and analytical fit (Eq. (10)) for: (a) hybridpassivated CQD solar cell and (b) organically cross-linked organic CQD solar cell.

Here, voltage-dependent losses occur due to the reduction in the electric field, resulting in longer transit times, for charge carriers transiting the thickness of the device. This model has been applied successfully to amorphous Silicon ${ }^{11}$ and $\mathrm{CdTe}^{7}$ material systems. It assumes that (1) the electric field is uniform in space within the depletion region; (2) this field extends throughout the entire thickness of the device; (3) diffusion makes a negligible contribution to photocurrent. This model works particularly well for $\mathrm{p}-\mathrm{i}-\mathrm{n}$ structures in which most absorption occurs in an intrinsic region through which the electric field extends.

While these previous models have been sufficient to describe the specific systems noted above, they fail to apply in thin-film p-n junction devices (Figure 2(c)). In these devices, significant carrier losses can occur even if presence of drift transport is the primary charge transport mechanism in a fully depleted device. To take account of this quantitatively, the linear decrease in the electric field profile from the junction to the edge of the depletion, per the Poisson equation, ${ }^{12}$ should be taken into account. Additionally, the depletion region thickness of the $p-n$ junction decreases with increased forward bias according to
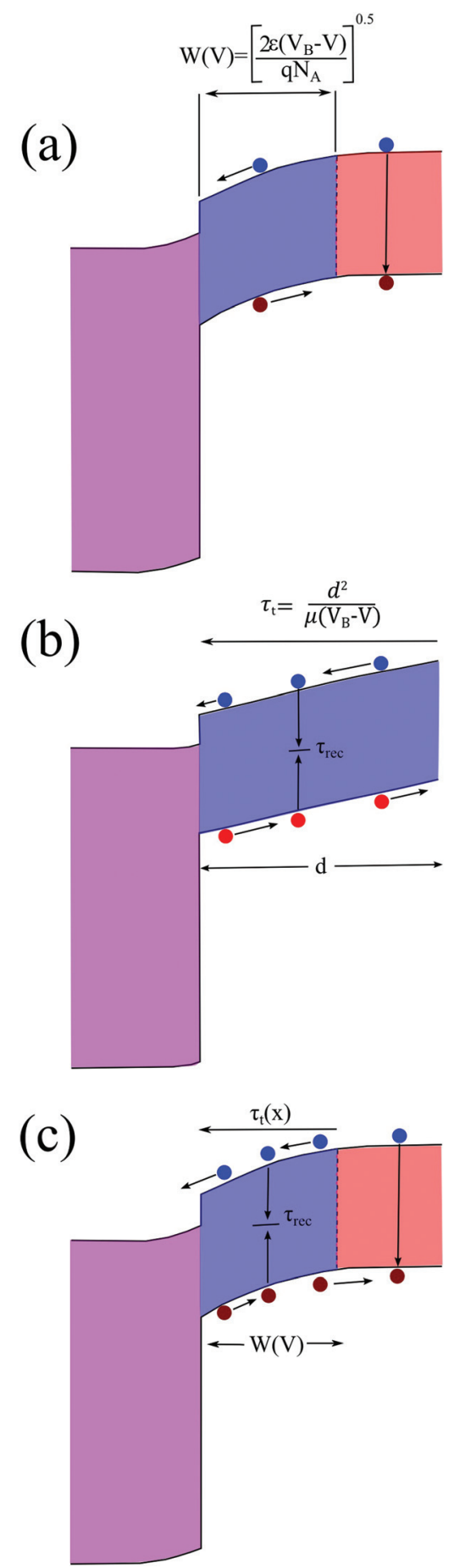

FIG. 2. Transport and recombination processes for (a) Gärtner p-n junction model (Eq. (2)); (b) Hecht drift transport model (Eq. (3)); and (c) proposed drift-limited p-n junction model (Eq. (10)).

$$
W(V)=\left[\frac{2 \varepsilon\left(V_{B}-V\right)}{q N_{A}}\right]^{0.5}=W(0)\left(V_{B}-V\right)^{0.5}
$$

where $\mathrm{W}(0)$ is the depletion region thickness at $0 \mathrm{~V}$ bias.

We therefore now proceed to derive a model that will apply to thin-film depleted bulk heterojunction CQD devices.

Transport of the minority carrier limits carrier extraction efficiency, as represented by the time for transport to the electron-accepting interfaces such as the n-type $\mathrm{TiO}_{2}$ interface in a CQD depleted heterojunction device. During this 
process, carriers may have the chance to recombine through Shockley-Read-Hall processes ${ }^{13}$ from trap-assisted and radiative recombination. In the linearly varying electric field, the electron accelerates as it moves through the device, and the transit time is given by

$$
\tau_{t}(x)=\int_{0}^{x} \frac{d x}{\mu E(x)+v_{D}},
$$

where $v_{\mathrm{D}}$ is the velocity of carriers undergoing diffusion

$$
v_{D}=\frac{L_{D}}{\tau_{\text {rec }}}=\sqrt{\frac{k T \mu}{q \tau_{\text {rec }}} .}
$$

The electric field within a p-n junction is provided by

$$
E(x)=\frac{2\left(V_{B}-V\right)}{W(V)}\left(1-\frac{x}{W(V)}\right) .
$$

The probability that a carrier at a given position is extracted for a given bias can be calculated from

$$
P(x)=\exp \left(\frac{-\tau_{t}(x)}{\tau_{r e c}}\right) .
$$

Letting the generation rate be relatively uniform throughout the device allows calculation of the bias-dependent collection efficiency by integrating over the entire device depletion region. This assumption applies particularly well at longer above-bandgap excitation wavelengths at which the absorption length $1 / \alpha$ is modest compared to the double-pass device thickness.

Integrating over the entire thickness of the device depletion region, we achieve

$$
\eta(V)=\left(\frac{1}{\kappa}\right)^{\gamma} \frac{1}{\gamma+1}\left[(\kappa)^{\gamma}-(\kappa-1)^{\gamma}\right],
$$

where

(a)

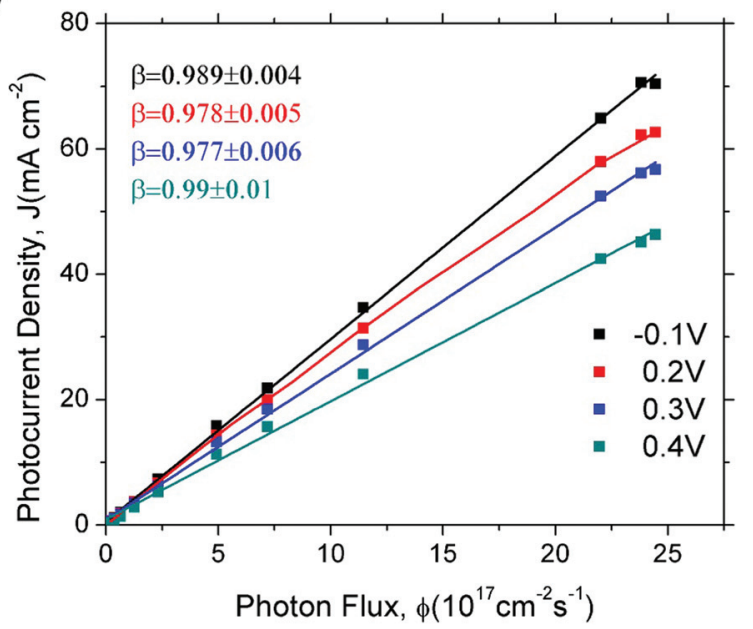

$$
\kappa=1+\frac{W(V) \sqrt{\frac{k T}{q \mu \tau_{r e c}}}}{2\left(V_{B}-V\right)}
$$

and

$$
\gamma=\frac{W(V)^{2}}{2\left(V_{B}-V\right) \mu \tau_{r e c}} .
$$

We fit the experimental data (Figures 1(b) and 1(c)) using this new model. Capacitance-voltage measurements indicate that devices remain fully depleted until $0.2 \mathrm{~V}$ forward bias. At this point, $\mathrm{W}(\mathrm{V})$ is equal to the device thickness d. By adjusting $\mu \tau_{\text {rec }}$, and thus the diffusion length, we were able to fit the experimental data well to the function of Eq. (10). Best fit values were obtained for diffusion lengths of $86 \mathrm{~nm}$ and $39 \mathrm{~nm}$, respectively. These values agree well with the $80 \mathrm{~nm}$ and $30 \mathrm{~nm}$ diffusion lengths obtained using donor-acceptor photoluminescence studies reported recently ${ }^{14}$ and confirm that the recent improvement in photovoltaic performance of CQD materials has been through enhancement in photocarrier transport lengths.

With the goal of elucidating further the dominant recombination processes in CQD solids, we examined intensity-dependent photocurrent as a function of forward bias (Figure 3(a)). The relationship between photocurrent density and intensity can provide information about the dominant recombination processes ${ }^{15}$

$$
J \propto I^{\beta}
$$

where $\mathbf{J}$ is the photocurrent density, $\mathrm{I}$ is the incident light intensity, and $\beta$ is the order of the power law relationship. Quantifying $\beta$ can offer insight into whether processes are monomolecular, bimolecular, or higher-order.

In Figure 3(b), the photocurrent varies linearly with intensity even at strong forward-bias voltages. This indicates that recombination losses occur principally through a firstorder process, such as trap-assisted recombination. Even at

(b)

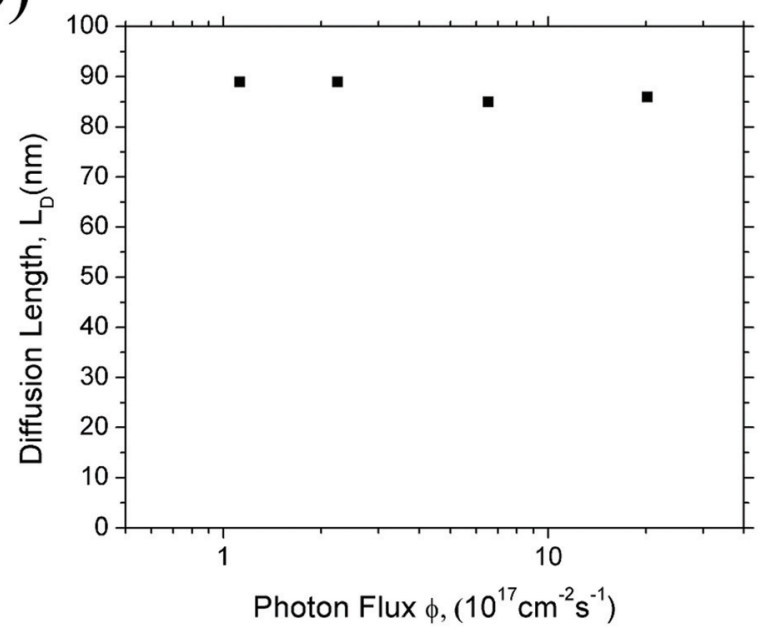

FIG. 3. (a) Intensity dependent photocurrent density for a hybrid passivated CQD solar cell. Linear lines of best fit are included to demonstrate unity power law relationship. (b) Intensity dependence of diffusion length for hybrid passivated CQD solar cells (Eq. (10)). 
forward bias, where carriers are less efficiently extracted and equilibrium carrier concentrations are greater than under short-circuit conditions, there is little evidence of higherorder recombination losses.

We applied our model to explore the intensitydependence of the diffusion length in the highest-performing CQD photovoltaic devices (Figure 3(b)). In the case of bimolecular recombination, we would expect to see a decrease in $\mathrm{L}_{\mathrm{D}}$ as the recombination rate increases under increased photoexcitation. No notable diminution in $\mathrm{L}_{\mathrm{D}}$ is seen as intensity is increased 20-fold. This agrees with the conclusion that bimolecular recombination is not a dominant loss mechanism in these devices and that trap-assisted recombination is likely the primary limiting factor for transport and, therefore, performance.

In summary, we have developed, from first principles, a model to describe carrier collection efficiency in CQD photovoltaics. The model assumes that carrier collection is largely driven by field-driven transport within the depletion region, with negligible contributions from a relatively small diffusion length. The model, which relied on a minimal set of initial assumptions, allowed us to determine the diffusion length accurately from measured J-V-L characteristics. We used this model to study previous reported CQD materials, finding that recent enhancements in performance have been the result of significant improvements in transport lengths.

We thank Angstrom Engineering and Innovative Technology for useful discussions regarding material deposition methods and control of glovebox environment, respectively. This publication is based on part of work supported by Award KUS-11-009-21, made by King Abdullah University of Science and Technology (KAUST), by the Ontario Research Fund Research Excellence Program, and by the Natural Sciences and Engineering Research Council (NSERC) of Canada.

${ }^{1}$ A. H. Ip, S. M. Thon, S. Hoogland, O. Voznyy, D. Zhitomirsky, R. Debnath, L. Levina, L. R. Rollny, G. H. Carey, A. Fischer, K. W. Kemp, I. J. Kramer, Z. Ning, A. J. Labelle, K. W. Chou, A. Amassian, and E. H. Sargent, Nat. Nanotechnol. 7, 577 (2012).

${ }^{2}$ G. I. Koleilat, I. J. Kramer, C. T. O. Wong, S. M. Thon, A. J. Labelle, S. Hoogland, and E. H. Sargent, Sci. Rep. 3, 2166 (2013).

${ }^{3}$ E. H. Sargent, Nat. Photonics 3, 325 (2009).

${ }^{4}$ R. Scheer and H.-W. Schock, in Chalcogenide Photovoltaics (Wiley-VCH Verlag GmbH \& Co. KGaA, Weinheim, Germany, 2011), pp. 9-127.

${ }^{5}$ R. A. Street, M. Schoendorf, A. Roy, and J. H. Lee, Phys. Rev. B 81, 205307 (2010).

${ }^{6}$ X. X. Liu and J. R. Sites, J. Appl. Phys. 75, 577 (1994).

${ }^{7}$ S. Hegedus, D. Desai, and C. Thompson, Prog. Photovoltaics 15, 587 (2007).

${ }^{8}$ W. W. Gärtner, Phys. Rev. 116, 84 (1959).

${ }^{9}$ I. J. Kramer, D. Zhitomirsky, J. D. Bass, P. M. Rice, T. Topuria, L. Krupp, S. M. Thon, A. H. Ip, R. Debnath, H.-C. Kim, and E. H. Sargent, Adv. Mater. 24, 2315 (2012).

${ }^{10}$ X. Lan, J. Bai, S. Masala, S. M. Thon, Y. Ren, I. J. Kramer, S. Hoogland, A. Simchi, G. I. Koleilat, D. Paz-Soldan, Z. Ning, A. J. Labelle, J. Y. Kim, G. Jabbour, and E. H. Sargent, Adv. Mater. 25, 1769 (2013).

${ }^{11}$ R. S. Crandall, J. Appl. Phys. 53, 3350 (1982).

${ }^{12}$ S. M. Sze and K. K. Ng, in Physics of Semiconductor Devices (John Wiley \& Sons, Inc., Hoboken, New Jersey, 2006), pp. 77-133.

${ }^{13}$ W. Shockley and W. T. Read, Phys. Rev. 87, 835 (1952).

${ }^{14}$ D. Zhitomirsky, O. Voznyy, S. Hoogland, and E. H. Sargent, ACS Nano 7, $5282(2013)$

${ }^{15}$ S. R. Cowan, J. Wang, J. Yi, Y.-J. Lee, D. C. Olson, and J. W. P. Hsu, J. Appl. Phys. 113, 154504 (2013). 